\title{
Numerical Analysis of Performance of Annular Fin
}

\author{
Salah EL-Badri \\ College of Mechanical Engineering Technology \\ Benghazi-Libya
}

\author{
Naser S. Sanoussi \\ College of Mechanical Engineering Technology \\ Benghazi-Libya
}

\author{
Gamal G. S. Hashem \\ Mechanical Engineering dept. \\ Faculty of Engineering, University of Benghazi \\ Benghazi-Libya
}

\begin{abstract}
The governing equation that represents the temperature distribution in annular fins is solved numerically. The finite difference technique is utilized in two dimensions under transient conditions. No angular variation of temperature is considered. The solution was conducted under the boundary conditions of insulated fin tip. The model was solved under practical ranges of different normalized parameters of radii ratio, $R_{l}$, aspect ratio, $A S R$ and Biot number, $B$ i.

The results in the form of fin efficiency are tested against a one-dimensional analytical solution. The comparisons showed good agreement at thin fins and less agreement at thick fins. This is expected since one dimensional solution is limited to thin fins. This agreement confirms that the current solution is accurate and reliable.

The results are generated and presented at different values of the above mentioned parameters. Generally the fin efficiency is shown to decrease with $B i$, and the $A S R$. However, the efficiency was shown to increase with $R_{l}$.
\end{abstract}

Keywords--Heat transfer; Annular fine; Fin performance; Temperature distribution

\section{INTRODUCTION}

Heat conducted through a solid substance is often transferred by convection to the surrounding fluid. Since the convection rate is proportional to the surface area, the heat dissipated can be increased by attaching strips of metal called "fins" to the surface, in the spaces where the large convection resistance occurs.

Fins are generally used on surfaces where the heat transfer coefficient is low. For any application, the problem is to choose a fin which will give a maximum cooling efficiency, minimum material for cost, weight, and space consideration, minimum resistance to the flow of the ambient medium, adequate strength and ease of manufacture. For this purpose detailed information on the performance of fins under different operating conditions and for different geometries is needed.

Fins are used to enhance convective heat transfer in a wide range of engineering applications, and offer a practical means for achieving a large total heat transfer surface area without the use of an excessive amount of primary surface area. Fins are commonly applied for heat management in electrical appliances such as computer power supplies or substation transformers. Other applications include IC engine cooling, such as fins in a car radiator. It is important to predict the temperature distribution within the fin in order to choose the configuration that offers maximum effectiveness.

Although there are many different situations that involve combined conduction-convection effects, the most frequent application is one in which an extended surface is used specifically to enhance the heat transfer rate between a solid and an adjoining fluid.

To illustrate the function of fins, consider the plane wall of Fig. 1a. If the fin surface temperature, $\boldsymbol{T}_{\boldsymbol{s}}$, is fixed, there are two ways by which the heat transfer rate may be increased. Either by increasing the convection coefficient, $\boldsymbol{h}$, by increasing the fluid velocity, and/or reducing the fluid temperature, $\boldsymbol{T}_{\infty}$. However; many situations would be encountered in which increasing $\boldsymbol{h}$ to the maximum possible value is either insufficient to obtain the desired heat transfer rate or the associated costs are prohibitive. Such costs are related to the blower or pump power requirements needed to increase $\boldsymbol{h}$ through increased fluid motion. Moreover, the second option of reducing $\boldsymbol{T}_{\infty}$ is often impractical. Examining Fig. 1b, however, we see that there exists a third option. That is, the heat transfer rate may be increased by increasing the surface area across which the convection occurs. This may be done by employing fins that extends from the wall into the surrounding fluid [1].

The thermal conductivity of the fin material has a strong effect on the temperature distribution along the fin and therefore influences the degree to which the heat transfer rate is enhanced. Ideally, the fin material should have a large thermal conductivity to minimize temperature variations from its base to its tip. In the limit of infinite thermal conductivity, the entire fin would be at the temperature of the base surface, thereby providing the maximum possible heat transfer enhancement, i.e. ideal fin.

A straight fin is any extended surface that is attached to a plane wall. It may be of uniform cross-sectional area, or its cross-sectional area may vary with the distance $\mathrm{x}$ from the wall. An annular fin is one that is circumferentially attached to a cylinder, and its cross section varies with radius from the centerline of the cylinder. 


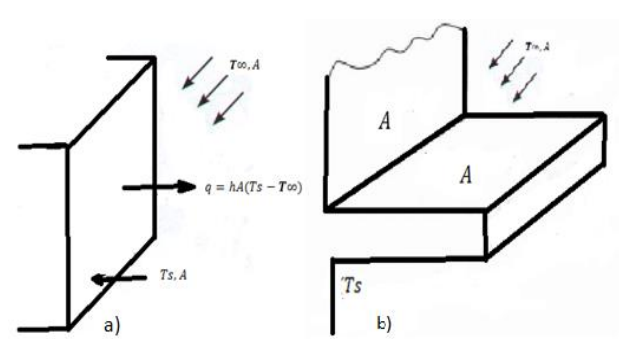

Fig.1 Illustration of fin performance

The foregoing fin types have rectangular cross sections, whose area may be expressed as a product of the fin thickness $t$ and the width $\mathrm{w}$ for straight fins or the circumference $\mathbf{2} \boldsymbol{\pi} \mathbf{r}$ for annular fins. In contrast a pin fin, or spine, is an extended surface of circular cross section. Pin fins may also be of uniform or non-uniform cross section. In any application, selection of a particular fin configuration may depend on space, weight, manufacturing, and cost considerations, as well as on the extent to which the fins reduce the surface convection coefficient and increase the pressure drop associated with flow over the fins.

However, Fins may be classified as straight surface area such as the trapezoidal fin and fins with curved surface area such as the parabolic and the cylindrical fins.

The aim of this study is to numerically study the performance of the annular fins. This study assumes two dimensional variations of temperature thus covering wider range of fin sizes with good accuracy. Our study also studies the performance variations with time.

\section{Nomenclature}

$\left(\mathrm{a}_{\mathrm{E}}, \mathrm{a}_{\mathrm{N}}, \mathrm{a}_{\mathrm{S}}, \mathrm{a}_{\mathrm{w}}, \mathrm{ap}_{\mathrm{P}}\right)$ ASR

b

$\mathrm{Bi}$

e

ETAT

Fo

$\mathrm{h}$

$\mathrm{k}$

$\mathrm{L}$

$\mathrm{M}$

n

N

$\mathrm{P}$

q

qact.

qideal

$\mathrm{r}$

$\mathrm{R}$

$\mathrm{R}_{1}$

$\mathrm{r}_{1}$

$\mathrm{r}_{2}$

$\mathrm{S}$

SS

$\mathrm{t}$

$\mathrm{T}$

$\mathrm{T}_{\mathrm{b}}$
Algebraic equation coefficient

Aspect ratio

Wall coefficient

Biot number

East control volume interface

Fin efficiency

Fourier number

Average convection heat transfer coefficient

Thermal conductivity

Fin length

Number of grid points along $(\mathrm{R})$

North control volume interface

Number of grid points along $(\xi)$

Central control volume node

Heat transfer rate

Actual heat transfer

Ideal heat transfer

Dimensional radial coordinate

Dimensionaless radial coordinate

Ratio of radius

Inner radius of fin

Outer radius of fin

South control volume interface

Fo at steady state

Time

Dimensional temperature

Fin base temperature
$\mathrm{T}_{\infty}$

W

$\mathrm{X}$

Greek symbols

a

$\delta$

$\Delta \mathrm{Fo}$

$\Delta \mathrm{R}$

$\Delta \xi$

$\theta_{\mathrm{E}}, \theta_{\mathrm{W}}, \theta_{\mathrm{S}}, \theta_{\mathrm{N}}$

$\theta \mathrm{P}$

$\theta \mathrm{P}$

$\xi$

\section{STATEMENT OF THE PROBLEM}

\section{A. Physical Model}

The geometry under consideration is an annular fin; the fin is characterized by its total length, L, its thickness, $2 \delta$, its inside radius $r_{1}$, and its outside radius, $r_{2}$. The fin material is assumed to be homogeneous with constant thermal conductivity equals to, $\mathrm{k}$. The fin skin is assumed to be totally surrounded by a fluid at a uniform temperature equals to, $\mathrm{T}_{\infty}$, with an average convective heat transfer coefficient equals to, h. The fin tip is assumed to be perfectly insulated, i.e. all heat dissipated from the fin is from the circular two sides of the fin. The temperature within the fin is assumed to be two dimensional in the radial and transverse directions and changes with time, i.e. transient.

The variations in temperature in the angular direction, $\Phi$ are neglected and assumed constant at each instant of time. Fig. 2 shows the annular fin under study with all involved parameters.

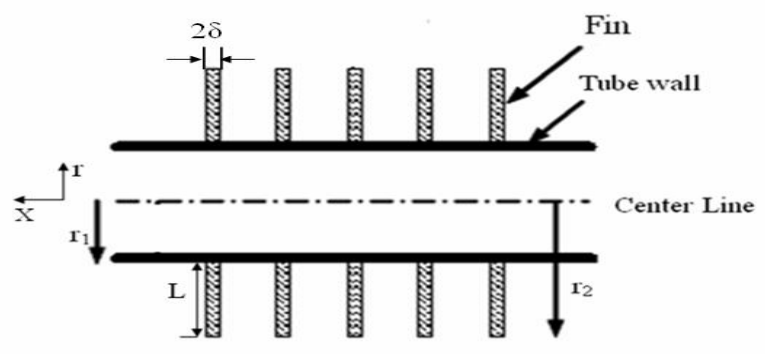

Fig. 2 Schematic diagram of annular fin

\section{B. Solution Domain}

Due to symmetry around an axis that extends radially and divides the fin into equal halves each with thickness equals to $\boldsymbol{\delta}$, and since there are no temperature variations in the angular direction, the solution domain is reduced to a rectangular area. This rectangular area extends axially from $\mathrm{x}=0$ at the symmetry line to $\mathrm{x}=\mathrm{d}$ at the fin surface, and it extends radially from $r=r_{1}$ at the fin base to $r=r_{2}$ at the fin tip, as can be seen in Fig. 3. 


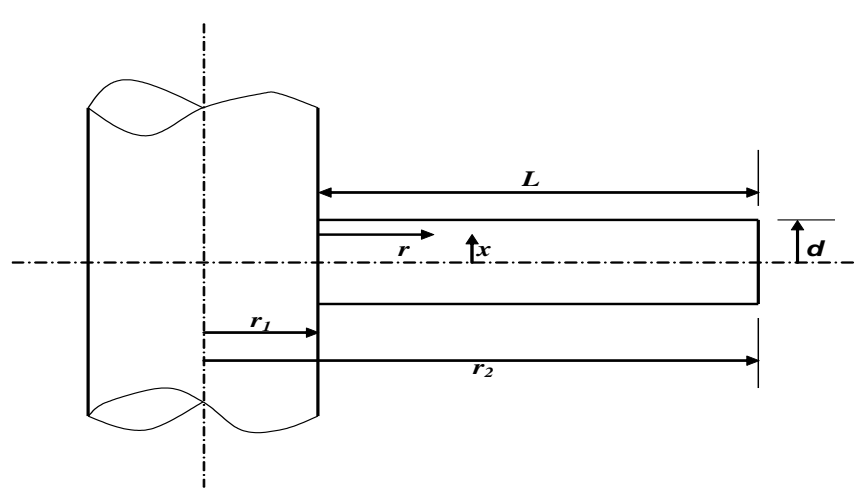

Fig. 3 Solution domain and coordinate system

\section{Mathematical Model}

The general conduction equation in cylindrical coordinate is given by the equation:

$$
\frac{\partial^{2} \boldsymbol{T}}{\partial r^{2}}+\frac{1}{r} \frac{\partial \boldsymbol{T}}{\partial r}+\frac{\partial^{2} \boldsymbol{T}}{\partial \boldsymbol{x}^{2}}+\frac{\partial^{2} \boldsymbol{T}}{\partial \phi^{2}}+\frac{q_{G}^{\cdots}}{k}=\frac{1}{\alpha} \frac{\partial \boldsymbol{T}}{\partial t}
$$

This equation is derived by applying the conservation principle to the differential element defined in cylindrical coordinates. Consider the two dimensional and no heat generation, homogeneous differential equation of heat conduction in the cylindrical system, the governing equation is reduced to:

$$
\frac{\partial^{2} T}{\partial r^{2}}+\frac{1}{r} \frac{\partial T}{\partial r}+\frac{\partial^{2} T}{\partial x^{2}}=\frac{1}{\alpha} \frac{\partial T}{\partial t}
$$

\section{Boundary Conditions and Initial Condition}

$$
\begin{array}{lll}
\text { At } & r=r_{1}, & T=T_{b} \\
& r=r_{2}, & \frac{\partial T}{\partial r}=\mathbf{O} \\
\text { At } & r=0, & \frac{\partial T}{\partial x}=\mathrm{O} \\
\text { At } & x=\delta, & -k \frac{\partial T}{\partial x}=h\left[T(\delta)-T_{\infty}\right] \\
\text { At } & x=0, & T(x, r, O)=T_{\infty} \\
\text { At } & t=0
\end{array}
$$

To normalize the mathematical model mentioned above with all boundary conditions and initial condition, the following parameters are used:

Dimensionless radial coordinate

$$
R=\frac{r}{r_{2}}
$$

Dimensionless axial coordinate

$$
\xi=\frac{x}{\delta}
$$

Dimensionless temperature

$$
\theta=\frac{T_{(r, x, t)}-T_{\infty}}{T_{b}-T_{\infty}}
$$

Dimensionless Time, (Fourier number),

$$
F_{o}=\frac{\alpha t}{\delta^{2}}
$$

The governing equation in dimensionless form is found by substituting equations (8) to (11) into equation (2) which gives:

$$
\frac{\partial^{2} \theta}{\partial R^{2}}+\frac{1}{R} \frac{\partial \theta}{\partial R}+A S R^{2} \frac{\partial^{2} \theta}{\partial \zeta^{2}}=A S R^{2} \frac{\partial \theta}{\partial F_{0}}
$$

Where;

$$
A S R=\frac{r_{2}}{\delta}
$$

The boundary conditions and the initial condition in the dimensionless form become:

$$
\begin{array}{ll}
\text { At } \quad \boldsymbol{R}=\boldsymbol{R}_{1}, \theta=1.0 \\
\text { At } \boldsymbol{R}=\mathbf{1}, \quad \frac{\partial \theta}{\partial R}=0 \\
\text { At } \xi=\mathbf{O}, \frac{\partial \boldsymbol{O}}{\partial \xi}=\mathbf{O} \\
\text { At } \xi=\mathbf{1}, \frac{\partial \theta}{\partial \xi}=-B i \theta(\delta) \\
\text { At } F_{o}=0 \quad, \quad \boldsymbol{\theta}(\zeta, R, 0)=0
\end{array}
$$

Where:

the Biot Number is

$$
B i=\frac{h \delta}{k}
$$

\section{METHOD OF SOLUTION}

The problem of determination of heat transfer through a fin requires the knowledge of temperature distribution in the fin, which in turn requires the solution of mathematical model and the applicable boundary conditions and initial condition.

There are four available methods for the evaluation of the temperature distribution; analytical, graphical, numerical and experimental Method

\section{A. Proposed Method of Solution}

Our proposal is to use the numerical method which is based on finite differences, and therefore it is an approximation computational technique. This method is preferred because it is straightforward, flexible, and it will frequently give a good approximate solution to a problem which its solution is complicated by formal analytical means.

\section{B. Control-volume formulation}

This method includes the tasks of providing a set of algebraic equations for these unknowns and of prescribing an algorithm for solving the equations. This control-volume formulation can be regarded as a special version of the method of weighted residuals. The calculation domain is divided into a number of non-overlapping control volumes such that there is one control volume surrounding each grid point as in the Fig.4. The differential equation is integrated over each control volume. 
The result is the discretization equation containing the values of $\theta$ for a group of grid points. The discretization equation obtained in this manner expresses the conservation principle for $\theta$ for the finite control volume, just as the differential equation expresses it for an infinitesimal control volume.

Deriving the control volume discretization equation by integrating the differential equation over a finite control volume is a rather roundabout process; the control volume equation would have been our only way of stating the conservation principle.

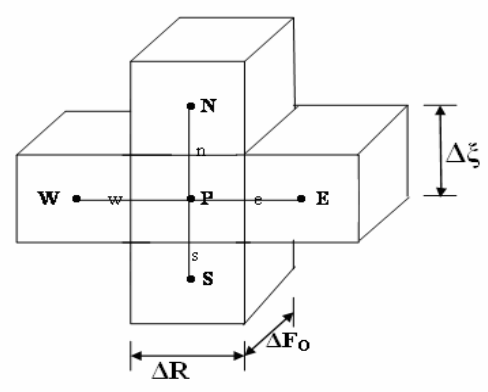

Fig.4 Three-dimensional cluster

\section{Solution Grid}

Due to symmetry between upper surface and lower surface in $\boldsymbol{x}$ direction of the annular fin, and since there is no variation in the temperature in the angular direction, the solution domain becomes a two dimensional grid. Fig. $5^{P}$

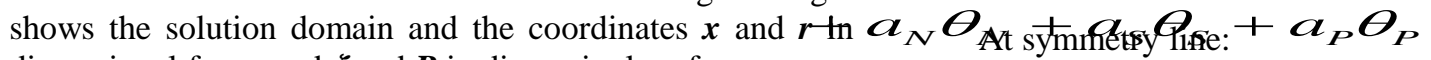
dimensional form, and $\boldsymbol{\zeta}$ and $\boldsymbol{R}$ in dimensionless form.

In the $\boldsymbol{r}$ direction the domain covers from $\boldsymbol{r}=\boldsymbol{r}_{\boldsymbol{I}}$ (the fin base) to $\boldsymbol{r}=\boldsymbol{r}_{2}$ (the fin tip), while in the $\boldsymbol{x}$ direction it covers from $\boldsymbol{x}=\boldsymbol{O}$ (the fin symmetry line) to $\boldsymbol{x}=\boldsymbol{\delta}$ (the fin surface). In dimensionless form, the solution domain covers from $\boldsymbol{R}=\boldsymbol{R}_{1}$ to $\boldsymbol{R}=\mathbf{1}$ in the radial direction and from $\zeta=\boldsymbol{0}$ to $\zeta=1$ in the axial direction.

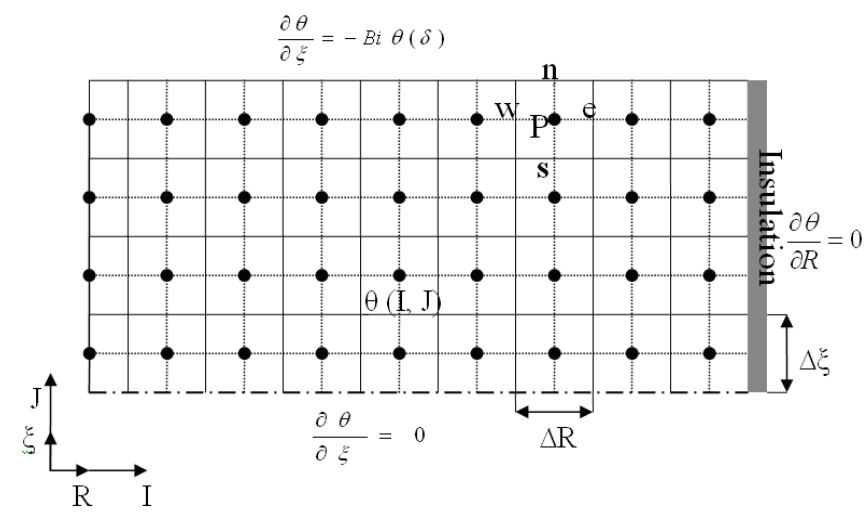

Fig. 5 Solution Grid

\section{Discretization of the Governing Equation}

To change the applicable mathematical model, boundary conditions, and initial condition into algebraic form, the approach of the control volume presented by Patankar [2] is used. Each term of the differential equation (12) is integrated over the limits of the boundary of the control volume for a single node as shown in the cluster of Fig. 4. The integration is performed as follows:
Integrating the first term of equation (12) as follows:

$$
\left.\int_{R} \int_{\xi} \int_{F_{o}} \frac{\partial^{2} \boldsymbol{\theta}}{\partial R^{2}} d F_{o} d \xi d R+\frac{\theta_{E}+\theta_{W}-2 \theta_{P}}{\Delta R}\right) \Delta F_{0} \Delta \zeta
$$

Similarly, the second term becomes:

$$
\int_{R} \int_{\xi} \int_{F_{o}} \frac{1}{R} \frac{\partial \theta}{\partial R} d F_{o} d \xi d R=\left(\frac{\theta_{E}-\boldsymbol{O}_{W}}{2 R}\right) \Delta F_{0} \Delta \zeta
$$

And the third term becomes:

$$
\begin{aligned}
\int_{R} \int_{\xi} \int_{F_{o}} \frac{r_{2}^{2}}{\delta^{2}} \frac{\partial^{2} \theta}{\partial \xi^{2}} d F_{o} d \xi d R= \\
\frac{r_{2}^{2}}{\delta^{2}}\left(\frac{\theta_{N}+\theta_{S}-2 \theta_{P}}{\Delta \zeta}\right) \Delta F_{0} \Delta R
\end{aligned}
$$

And the fourth term becomes:

$$
\int_{R} \int_{\xi} \int_{F_{o}} \frac{r_{2}^{2}}{\delta^{2}} \frac{\partial \theta}{\partial F_{o}} d F_{o} d \xi d R=\frac{r_{2}^{2}}{\delta^{2}}\left(\theta_{p}^{\backslash}-\theta_{p}\right) \Delta \zeta \Delta R
$$

Substituting by the four equations into the main equation (12), we obtain:

$$
\theta_{P}^{\prime}=\left(a_{E} \theta_{E}+a_{W} \theta_{W}+a_{N} \theta_{N}+a_{P} \theta_{P}\right) /\left(1-a_{S}\right)
$$

At the fin surface:

$$
\theta_{P}^{\prime}=\left(a_{E} \theta_{E}+a_{W} \theta_{W}+a_{S} \theta_{S}+a_{p} \theta_{P}\right) /\left(1-b^{*} a_{N}\right)
$$

At the fin tip:

$$
\theta_{P}^{\prime}=\left(a_{W} \theta_{W}+a_{N} \theta_{N}+a_{S} \theta_{S}+a_{P} \theta_{P}\right) /\left(1-a_{E}\right)
$$

At the corner of the symmetry line and tip:

$$
\theta_{P}^{\prime}=\left(a_{W} \theta_{W}+a_{N} \theta_{N}+a_{P} \theta_{P}\right) /\left(1-a_{E}-a_{S}\right)
$$

At the corner of the surface and the tip:

$$
\theta_{P}^{\prime}=\left(a_{W} \theta_{W}+a_{S} \theta_{S}+a_{P} \theta_{P}\right) /\left(1-a_{E}-b * a_{N}\right)
$$

Where:

$$
\begin{gathered}
a_{E}=\left(\frac{1}{\Delta R}+\frac{1}{2 R}\right) \frac{1}{A S R^{2}} \frac{\Delta F_{0}}{\Delta R} \quad, \quad a_{W}=\left(\frac{1}{\Delta R}-\frac{1}{2 R}\right) \frac{1}{A S R^{2}} \frac{\Delta F_{0}}{\Delta R} \\
a_{N}=\frac{\Delta F_{o}}{\Delta \xi^{2}} \quad, \quad a_{S}=\frac{\Delta F_{o}}{\Delta \xi^{2}} \\
a_{P}=1-\frac{1}{A S R^{2}} \frac{2 \Delta F_{0}}{\Delta R^{2}}-\frac{2 \Delta F_{0}}{\Delta \zeta^{2}} \quad, \quad b=\frac{2-B i * \Delta \xi}{2+B i * \Delta \xi}
\end{gathered}
$$




\section{E. Solution Procedure}

Equations (20) to (25) are applied each at its appropriate location and the solution is attained by a point by point Gauss-Seidel iteration method. The iteration continues until a convergence is reached. This convergence is measured by a criterion of the form $|(\boldsymbol{\theta}-\boldsymbol{\theta p}) / \boldsymbol{\theta}| \leq 10-5$ is achieved at each nodal point. Where $\boldsymbol{\theta}$ is the temperature at the present iteration and $\boldsymbol{\theta p}$ is the temperature of the previous iteration at the same node.

Once a convergence is reached, the solution is progressed to the next time step and continues until steady state conditions are reached.

\section{F. Fin Performance Calculation}

There are several ways used for measuring the relative performance of fin. The first of these is effectiveness, which is the heat conducted through the fin base to that which would be transferred from the same base area if the fin were not present, and if the base temperature ( $\mathbf{T b}$ ) remains the same, but this criterion is not a true indication of fin performance, since (Tb) cannot be expected to remain constant if the fin is removed. A second and more realistic measure of fin performance is the ratio of the actual heat dissipated by the fin to that which would be dissipated if the entire fin temperatures were at (Tb), (i.e. ideal fin). This ratio, if calculated under steady state conditions, is known as the fin efficiency.

In this work we use the second measure as an index of performance and refer to it as the fin efficiency.

\section{G. Calculation of Heat Transfer Parameters}

Since the tip is insulated, the heat loss from the fin tip does not exist and all heat loss is that from the lateral surface.

The actual heat transfer distribution $\boldsymbol{q}_{\text {act }}$ from the fin surface area is calculated locally at each radial location, $\boldsymbol{r}$ covering a surface area in the form of a ring equals to $(2 \mathrm{pr} \Delta r)$.

This actual heat transfer through the fin surface is given by:

$$
q_{\text {act } .}=\frac{-k}{\delta}(2 \pi r \Delta r)\left(T_{b}-T_{\infty}\right) \frac{\partial \theta}{\partial \zeta}
$$

And the ideal heat transfer through the entire fin surface area assuming that the whole fin is isothermal at the base temperature $\boldsymbol{T} \boldsymbol{b}$ is given by:

$$
q_{\text {ideal }}=h \pi\left(r_{2}^{2}-r_{1}^{2}\right)\left(T_{b}-T_{\infty}\right)
$$

From Equation (26) and (27) we get:

$$
q=\frac{q_{\text {act }}}{q_{\text {ideal }}}=-\frac{1}{B_{i}} \frac{2 R \Delta R}{\left(1-R_{1}^{2}\right)} \frac{\partial \theta}{\partial \zeta}
$$

From boundary condition at $\zeta=1$ we have $\frac{\partial \theta}{\partial \zeta}=-B i \theta(\delta)$

Then equation (28) can be written in the form:

$$
q=\frac{q_{\text {act } .}}{q_{\text {ideal }}}=\frac{2 R \Delta R}{\left(1-R_{1}^{2}\right)} \theta(\delta)
$$

To get the fin efficiency, we need to calculate the ratio of the total actual heat transfer from the fin to the ideal heat loss from the same fin geometry. This can be achieved by numerically integrating equation (29) over the whole fin surface

$$
E T A T=\frac{2 \Delta R}{\left(1-R_{1}^{2}\right)} \sum_{R=R 1}^{R=1}[R \theta(\delta)]
$$

Where: $\boldsymbol{E T A T}$ is the fin efficiency.

\section{H. Selection of Mesh Size}

The mesh size was selected on the basis of many trials. Due to the nature of the problem, a huge memory is needed to store the temperature and the finer the mesh the greater the memory needed. The execution time was also another factor that played a major role in selecting a reasonable mesh size.

Our aim is to select a mesh size that gives reasonably accurate solution with optimized execution time and memory size. Our final selection of mesh size was as follows:

40 equally spaced mesh points in the radial direction.

20 equally spaced mesh points in the axial direction.

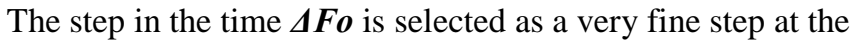
fin base equals to $\mathbf{0 . 0 0 0 5}$. This value then increased gradually as we march in the time space by multiplying it by the factor 1.01 until $\Delta F o$ reached a value of 0.001 then kept constant until we reach the steady state conditions.

The steady state conditions were assumed when the value of the total heat transfer from the fin, ETAT, as defined by equation (30), becomes reasonably constant with Fourier number, Fo. The criterion used to assume a steady state condition is to compare the value of $\boldsymbol{E T A T}$ with its value at the previous time step, $\boldsymbol{E T A T}$ as:

$\left|\frac{\boldsymbol{E T A} \boldsymbol{T}-\boldsymbol{E T A} \boldsymbol{T}_{p}}{\boldsymbol{E} \boldsymbol{T A T} T}\right| \leq \mathbf{1 0}^{-6}$, when this criterion is satisfied the solution is stopped and assumed complete.

The value of $\boldsymbol{F o}$ at which the steady state conditions are reached is called $\boldsymbol{F o s s}$, and the value of $\boldsymbol{E T A T}$ at which the steady state conditions are reached is the steady state fin efficiency.

\section{Validation of Accuracy}

To validate the accuracy of our solution, a comparison in the form of $\boldsymbol{E T A T}$ with $\boldsymbol{B} \boldsymbol{i}$ is presented against analytical one-dimensional solution as presented in Ref. [2]. This comparison is shown in Fig. 6.

The comparisons were made at two extreme geometrical cases, one with too thick and short fin (Minimum $\boldsymbol{A S R}$ and maximum $\boldsymbol{R}_{\boldsymbol{l}}$ ), and the other with too thin and long fin (Maximum $\boldsymbol{A S R}$ and minimum $\boldsymbol{R}_{\boldsymbol{l}}$ ). It can be seen clearly that the agreement with the thin and long fin is excellent, whereas the comparison with the thick and short fin has some deviations. These findings are expected since the assumption of one-dimensional solution of Ref. [2] is not expected to give accurate results when the fins are thick and short. Thus this validates the accuracy of our model, method of solution and selected mesh size. 


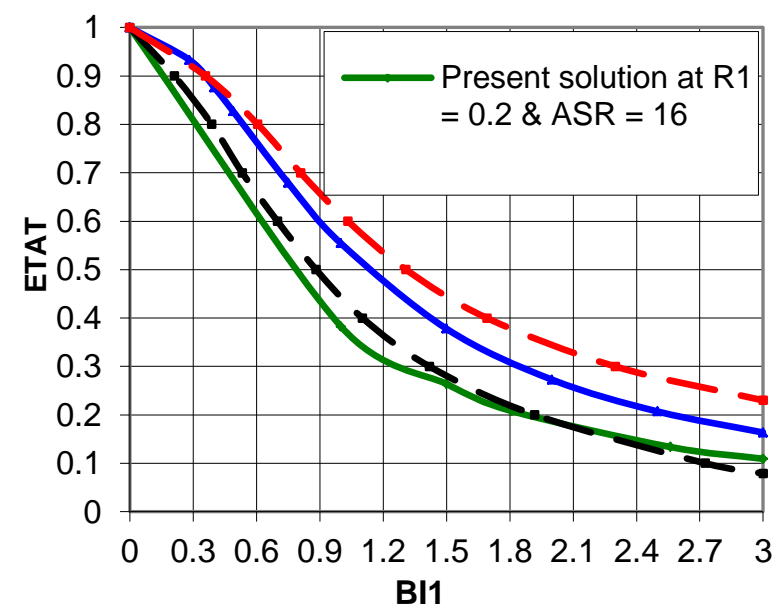

Fig. 6 Comparison of present solution with one dimension solution of [2]

\section{RESULTS AND DISCUSSION}

The result in the form of temperature distribution in the fin body and the heat transfer rate distribution over the fin surface in dimensionless form are present at different geometrical parameters of $\boldsymbol{A S R}$ and $\boldsymbol{R}_{\boldsymbol{I}}$ and different values of $\boldsymbol{B i}$ and $\boldsymbol{F o}$ ". The fin efficiency which is an important parameter is presented at different parameters to show the fin performance at different geometrical and operational parameters.

The range values of $\boldsymbol{A S R}$ covered in this study are 10,12 , 14, and 16, while the range of values of $R_{1}$ covered are 0.2 , 0.4, 0.6, and 0.8. The Biot number, $\boldsymbol{B} \boldsymbol{i}$ range covered in this study covers the values of $0,0.04,0.08$, and 0.12 . These ranges of parameters cover the practical and meaningful values that are generally encountered in practical life.

\section{A. Fin Efficiency}

The fin efficiency is an indication of how much energy a specific fin can dissipate when compared to an ideal fin of similar size. An ideal fin is a fin with zero conductive resistance within the fin material; the ideal fin dissipates the maximum amount of heat that can possibly be dissipated by a fin of that size. Mathematically when $\boldsymbol{B i}$ equals to zero, the fin becomes ideal. The ideal fin is characterized by its isothermal temperature throughout the fin body and equals to the temperature of the fin base, $\boldsymbol{T}_{\boldsymbol{b}}$.

In Fig.7, the fin efficiency, ETAT, is presented as a function of Biot number $\boldsymbol{B} \boldsymbol{i}$, at different values of $\boldsymbol{A S R}$ and $\boldsymbol{R}_{\boldsymbol{I}}$. It can be seen from the figure that as $\boldsymbol{B} \boldsymbol{i}$ increases the fin efficiency decreases. The rate of decrease is sharp at lower values of $\boldsymbol{B i}$. However; as $\boldsymbol{B i}$ increases to higher values the rate of decrease in efficiency becomes smaller. The values of the efficiency at any $\boldsymbol{B} \boldsymbol{i}$ are shown to be higher at higher values of $\boldsymbol{R}_{\boldsymbol{l}}$ and consistently lower at higher values of $\boldsymbol{A S R}$. This means that $\boldsymbol{R}_{\boldsymbol{I}}$ has the effect of increasing the efficiency while the $\boldsymbol{A S R}$ has the opposite effect of lowering the values of the efficiency at all values of $\boldsymbol{B i}$.

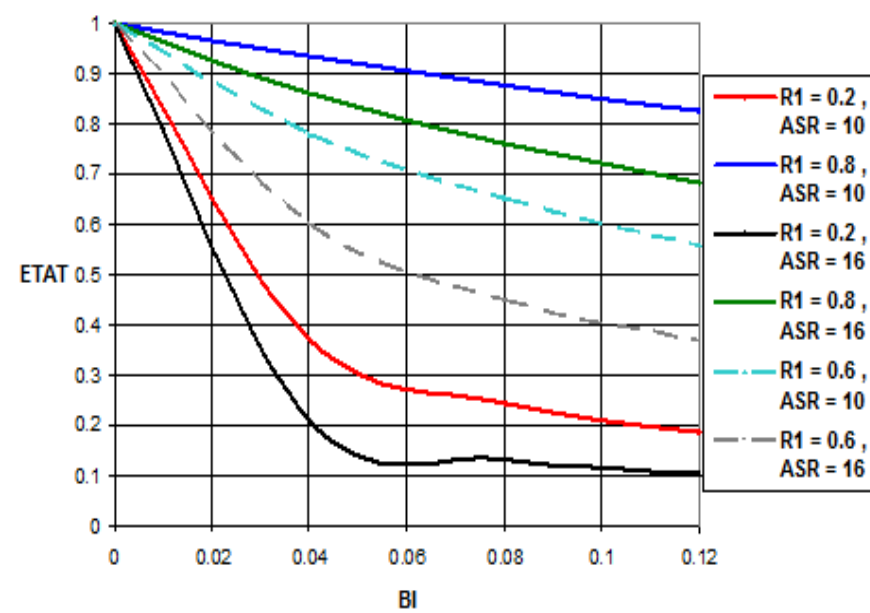

Fig. 7 Variation of fin efficiency with $B i$ at different values of $R_{1}$ and ASR

\section{B. Heat Transfer Rate Distribution}

The dimensionless heat transfer rate distribution, $\boldsymbol{q}$ along the radial direction, $\boldsymbol{R}$ is presented in eight figures. Fig. 8 to Fig. 11 represent the distribution of the heat transfer rate, $\boldsymbol{q}$ with $\boldsymbol{R}$ at different values of $\boldsymbol{B i}$ ranging from 0.04 to 0.12 . The first two figures, Fig. 8 and Fig. 9, are for minimum $\boldsymbol{A S R}$ of $\mathbf{1 0}$ while the other two figures of, Fig. 10 and Fig. 11, are for the maximum value of $\boldsymbol{A S R}$ of 16. The other four figures, from Fig. 12 to Fig. 15, show the heat transfer distribution, $\boldsymbol{q}$ with $\boldsymbol{R}$ at different values of $\boldsymbol{A S R}$ ranging from 10 to 16. The first two figures, Fig. 12 and Fig. 13, are for the minimum value of $\boldsymbol{B i}$ of $\mathbf{0 . 0 4}$ and the other two figures, Fig. 14 and Fig. 15, are for a maximum value of $B i=0.12$.

These eight figures in all show the effect of $\boldsymbol{A S R}, \boldsymbol{R}_{\boldsymbol{l}}$, and $\boldsymbol{B} \boldsymbol{i}$ on the heat transfer distribution along the fin surface. It can be said that the heat transfer rate decreases as $\boldsymbol{B i}$ increases. The effect of $\boldsymbol{R}_{\boldsymbol{l}}$ is shown to increase the value of the transfer rate at all values of $\boldsymbol{B} \boldsymbol{i}$ and $\boldsymbol{A S R}$. However; the effect of ASR is shown to have the opposite effect of decreasing the heat transfer rate at all values of $\boldsymbol{B} \boldsymbol{i}$ and $\boldsymbol{R}_{\boldsymbol{I}}$.

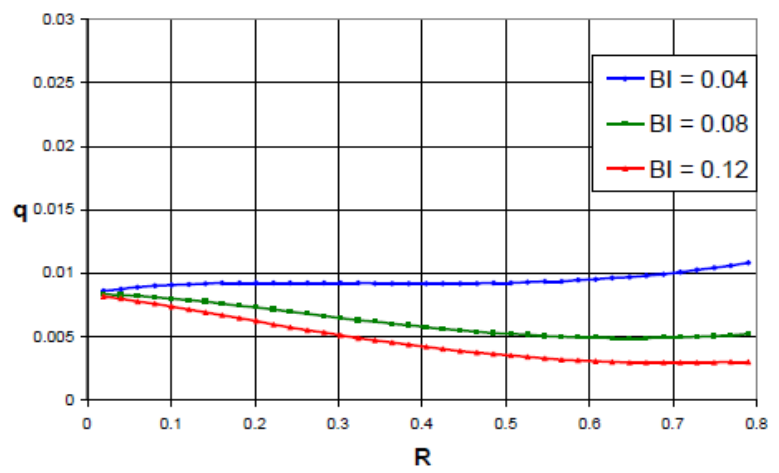

Fig. 8 Distribution of heat transfer rate at $\mathbf{A S R}=\mathbf{1 0}, \mathbf{R}_{\mathbf{1}}=\mathbf{0 . 2}$ and different values of $\mathbf{B i}$ 


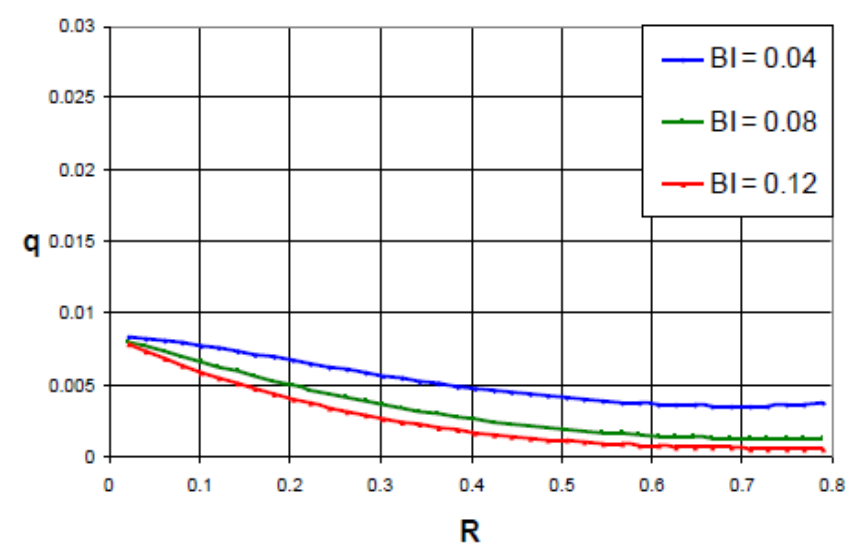

Fig. 9 Distribution of heat transfer rate at $\mathbf{A S R}=\mathbf{1 6}, \mathbf{R}_{\mathbf{1}}=\mathbf{0 . 2}$ and different values of $\mathbf{B i}$

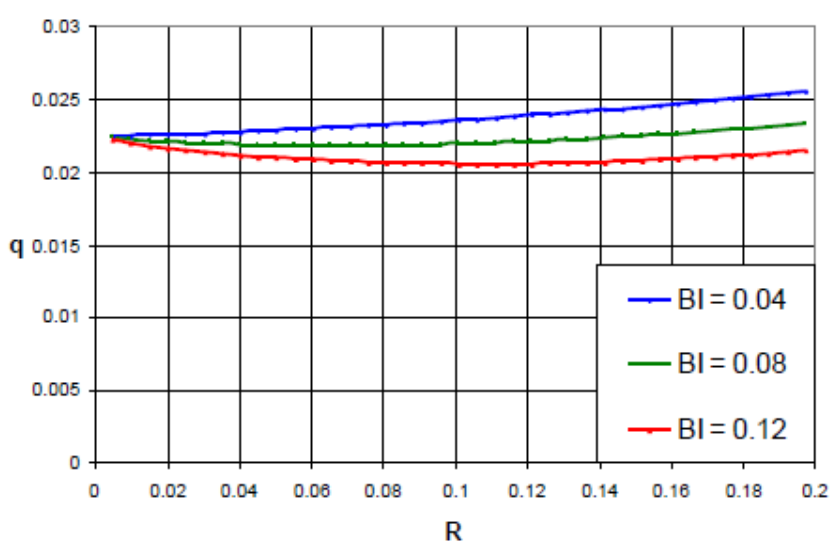

Fig. 10 Distribution of heat transfer rate at $\mathbf{A S R}=\mathbf{1 0}, \mathbf{R}_{\mathbf{1}}=\mathbf{0 . 8}$ and different values of $\mathbf{B i}$

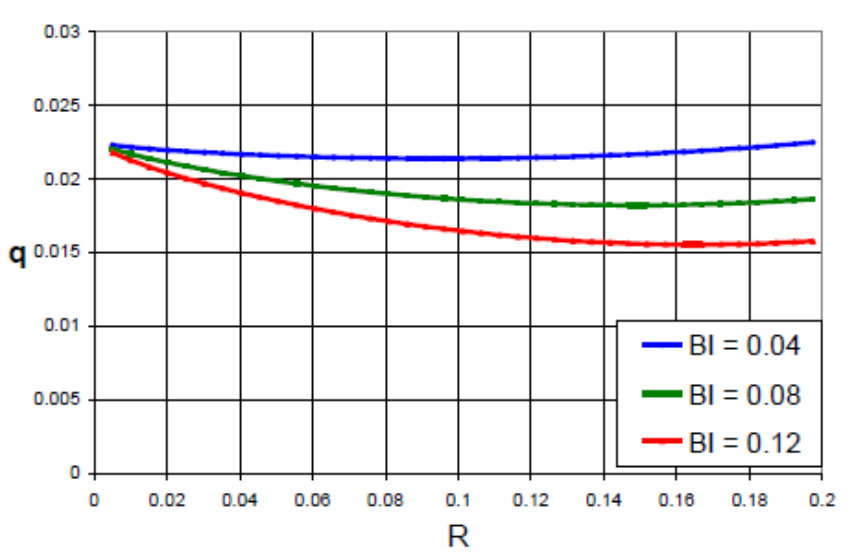

Fig. 11 Distribution of heat transfer rate at $A S R=16, R_{1}=0.8$ and different values of $\mathrm{Bi}$

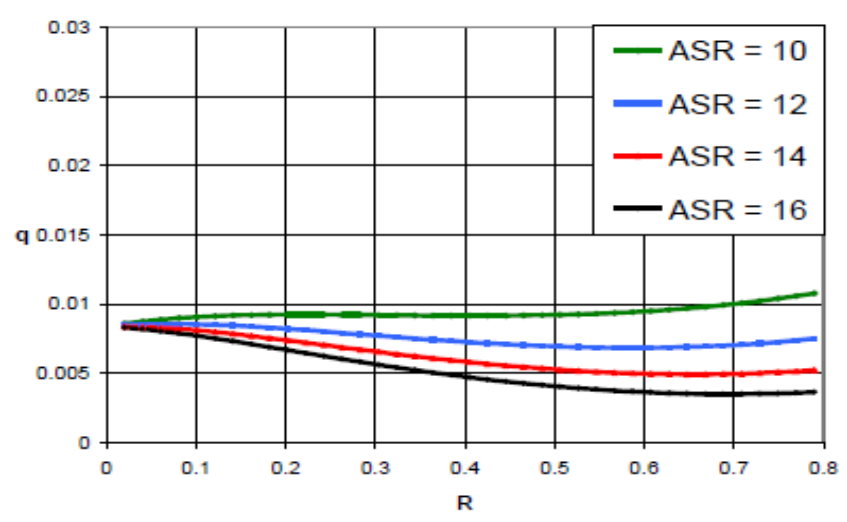

Fig. 12 Distribution of heat transfer rate at $\mathrm{Bi}=0.04, \mathrm{R}_{1}=0.2$ and different values of ASR

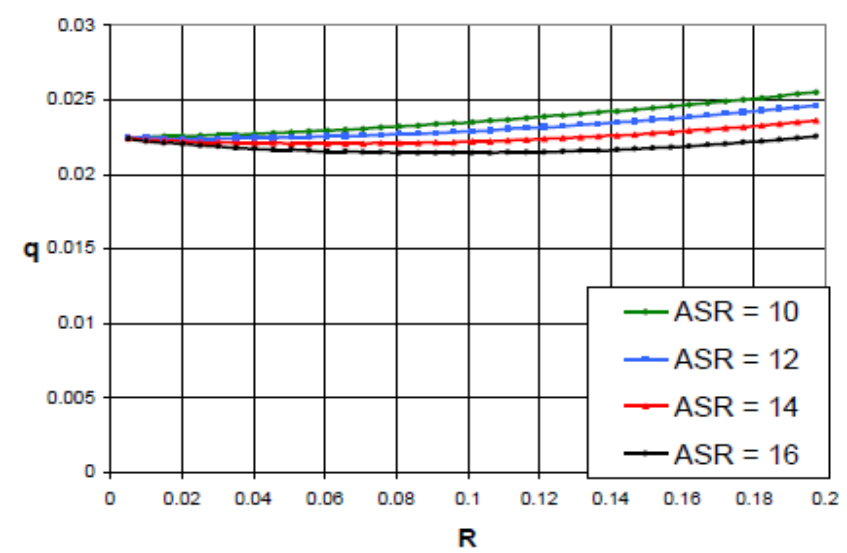

Fig.13 Distribution of heat transfer rate at $\mathbf{B i}=\mathbf{0 . 0 4}, \mathbf{R}_{\mathbf{1}}=\mathbf{0 . 8}$ and different values of ASR

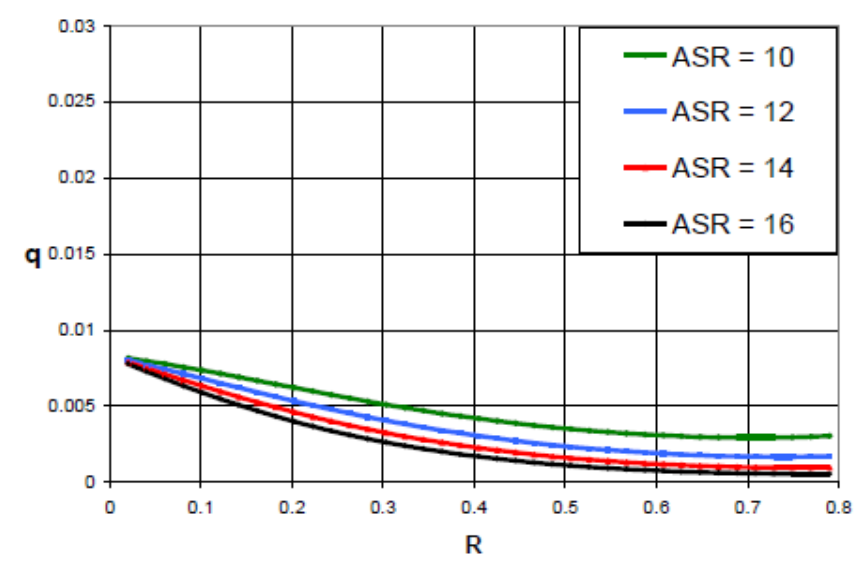

Fig. 14 Distribution of heat transfer rate at $\mathrm{Bi}=0.12, \mathrm{R}_{1}=0.2$ and different values of ASR 


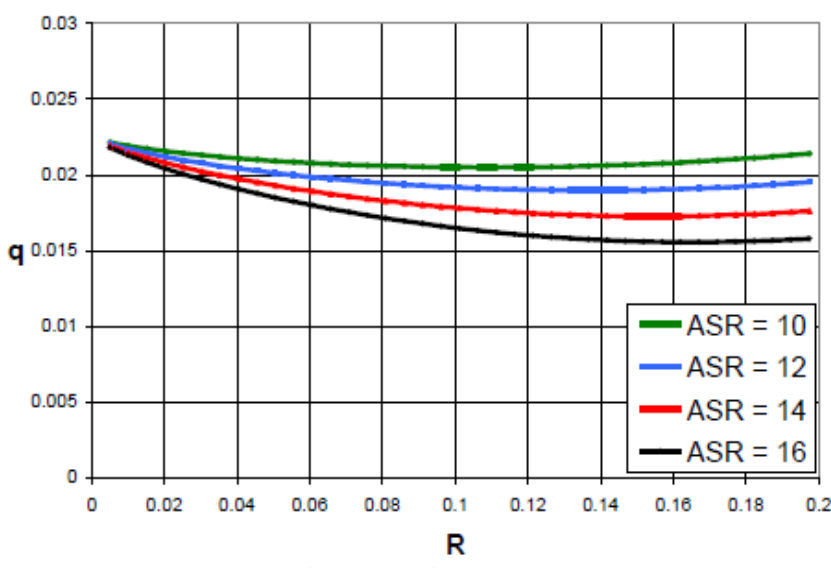

Fig. 15 Distribution of heat transfer rate at $B i=0.12, R_{1}=0.8$ and different values of ASR

Figures, from Fig. 16 to Fig. 19, show the heat transfer rate distribution with $\boldsymbol{R}$ at different values of $\boldsymbol{R}_{\boldsymbol{I}}$ and covering the limiting values of $\boldsymbol{A S R}$ and $\boldsymbol{B} \boldsymbol{i}$ by taking the minimum and maximum of each, as shown in the figures. The figures, Fig. 16 and Fig. 17, are for minimum value of $\boldsymbol{B} \boldsymbol{i}=\mathbf{0 . 0 4}$. Comparing these two figures we can conclude that the $\boldsymbol{R}_{\boldsymbol{I}}$ consistently increases the heat transfer rate while the $\boldsymbol{A S R}$ decreases the heat transfer rate. Similar findings can be drawn from the next two figures, Fig. 18 and Fig. 19, which are at the maximum value of $\boldsymbol{B} \boldsymbol{i}=\mathbf{0 . 1 2}$.

The general trend of the heat transfer rate with $\boldsymbol{R}$ has the tendency to decrease at early sections of $\boldsymbol{R}$ and attempt to increase at higher values of $\boldsymbol{R}$. This is attributed to the fact that as we move towards the fin tip the fin temperature decreases thus the heat transfer rate decreases; however, as we proceed towards the fin tip the surface area of the fin which is a function of $\boldsymbol{R}$ increases thus increases the heat transfer rate. With these two counter acting effects depending on which of the two effects dominates the rate of heat transfer decreases or increases.

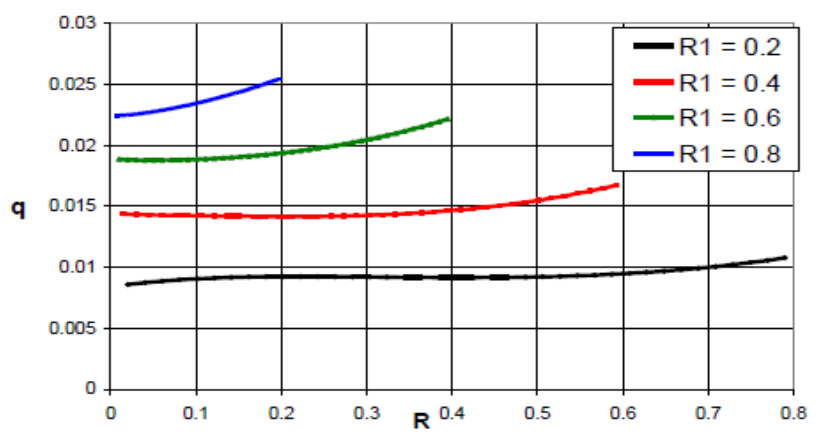

Fig. 16 Distribution of heat transfer rate at $\boldsymbol{B i}=\mathbf{0 . 0 4}, \boldsymbol{A S R}$ $=\mathbf{1 0}$ and different values of $\boldsymbol{R}_{\boldsymbol{I}}$

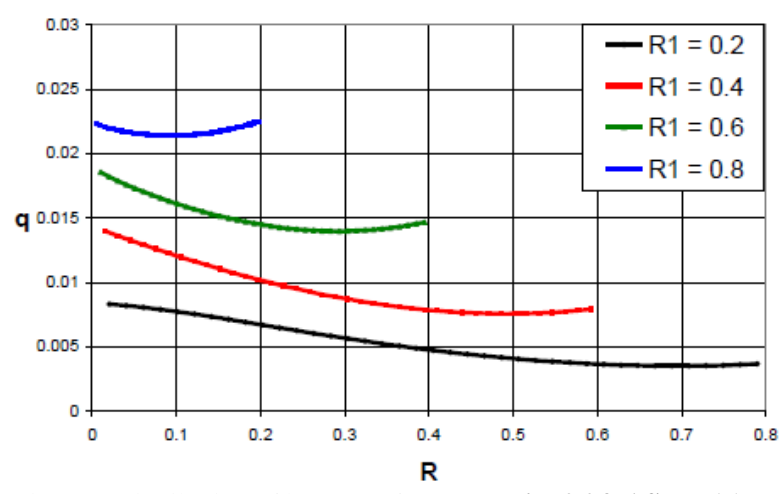

Fig. 17 Distribution of heat transfer rate at $\mathbf{B i}=\mathbf{0 . 0 4}, \mathbf{A S R}=\mathbf{1 6}$ and different values of $\mathbf{R}_{1}$

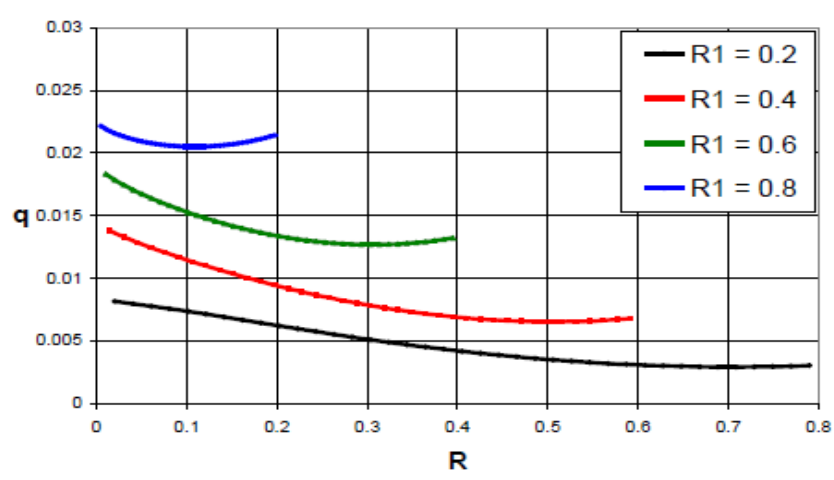

Fig. 18 Distribution of heat transfer rate at $\mathbf{B i}=\mathbf{0 . 1 2}, \mathbf{A S R}=\mathbf{1 0}$ and different values of $\mathbf{R}_{\mathbf{1}}$

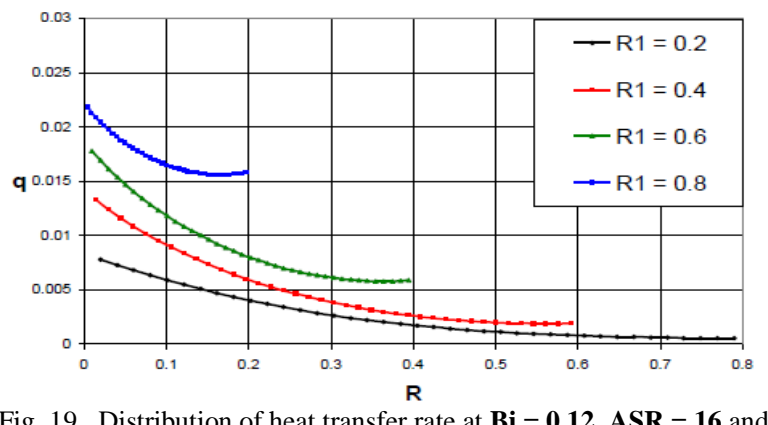

Fig. 19 Distribution of heat transfer rate at $\mathbf{B i}=\mathbf{0 . 1 2}, \mathbf{A S R}=\mathbf{1 6}$ and different values of $\mathbf{R}_{\mathbf{1}}$

\section{Effect of Fo on the Heat Transfer Rate Distribution}

To study the effect of $\boldsymbol{F o}$ on the distribution of heat transfer from the fins at different parameters, eight figures are presented. Figures, from Fig. 20 to Fig. 27, present the heat transfer rate distribution with $\boldsymbol{R}$ at different values of $\boldsymbol{F o}$, starting from small value of $\boldsymbol{F o}$ to the maximum value at the steady state. All figures show that at early stages of time, (low Fo) the heat transfer rate is very small and concentrates at areas close to the fin base. As $\boldsymbol{F o}$ increases, the heat transfer rate spreads further towards the fin tip. This is due to the fact that at smaller values of $\boldsymbol{F o}$, the fin is storing heat and only small portion is transferred to the surroundings. At the steady state value of $\boldsymbol{F o}$, all heat conducted into the fin through the base is dissipated by convection from the fin surface. Thus, the higher the value of $\boldsymbol{F o}$ the higher the heat transfer rate from the fin. 
Comparing these figures, it can be seen that higher values of $\boldsymbol{R}_{\boldsymbol{I}}$ increases the heat transfer rate distribution. However, both $\boldsymbol{B i}$ and $\boldsymbol{A S R}$ have an opposite effect on the heat transfer rate distribution for all values of $\boldsymbol{F o}$.

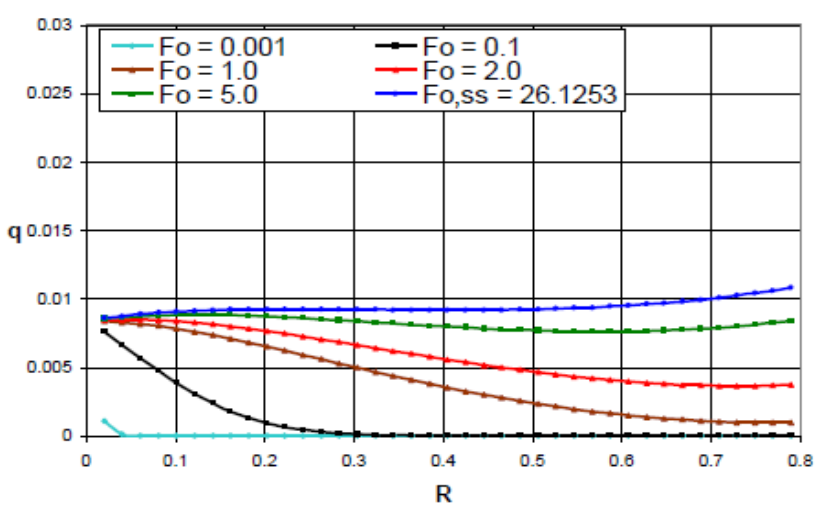

Fig. 20 Distribution of heat transfer rate at $\mathrm{R}_{1}=0.2, \mathrm{ASR}=10$, and $\mathrm{Bi}=$ 0.04 at different values of $F_{o}$

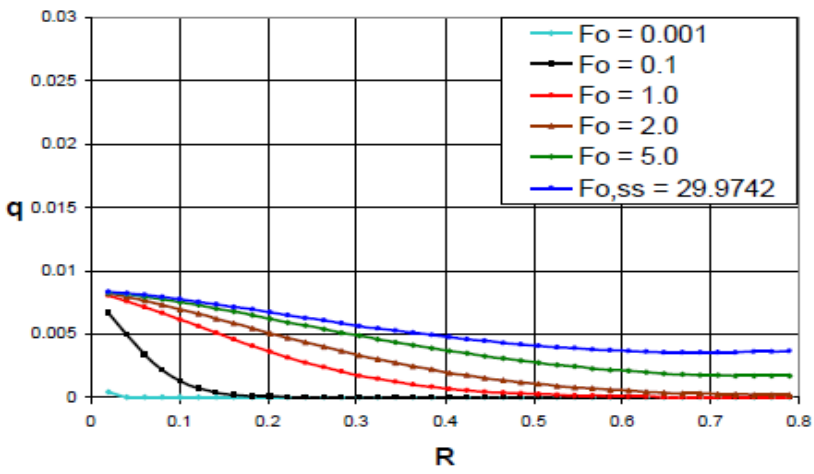

Fig. 21 Distribution of heat transfer rate at $\mathrm{R}_{1}=0.2, \mathrm{ASR}=16$, and $\mathrm{Bi}=0.04$ at different values of $\mathrm{F}_{\mathrm{o}}$

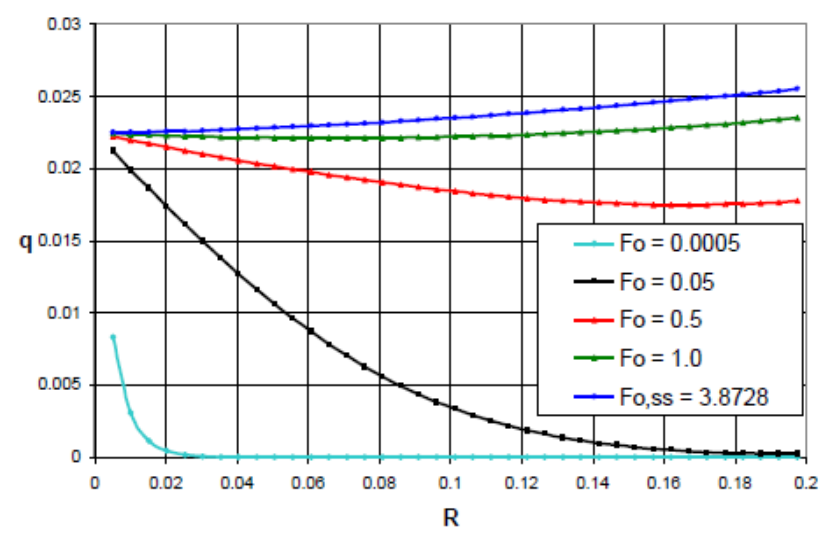

Fig. 22 Distribution of heat transfer rate at $\mathrm{R}_{1}=0.8, \mathrm{ASR}=10$, and $\mathrm{Bi}=$ 0.04 at different values of $F_{o}$

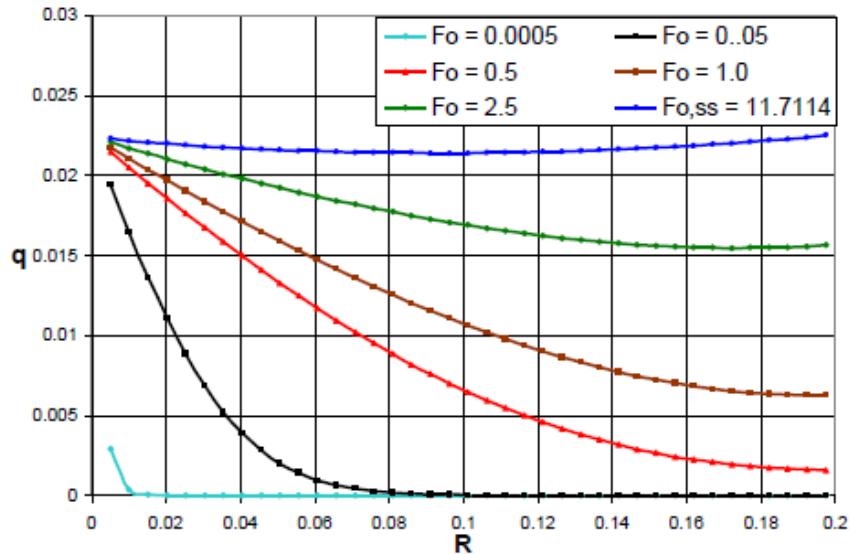

Fig. 23 Distribution of heat transfer rate at $\mathbf{R}_{\mathbf{1}}=\mathbf{0 . 8}, \mathbf{A S R}=\mathbf{1 6}$, and $\mathbf{B i}=$ $\mathbf{0 . 0 4}$ at different values of $\mathbf{F}_{\mathbf{0}}$

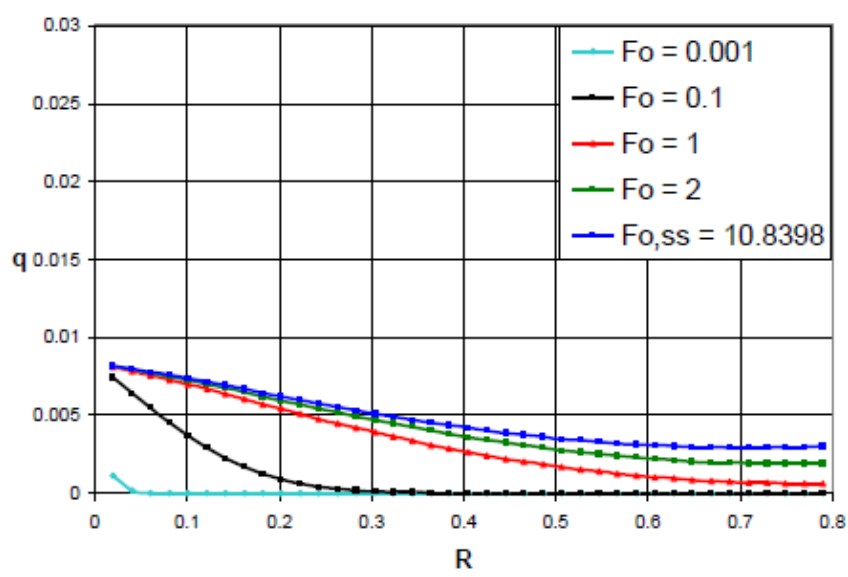

Fig. 24 Distribution of heat transfer rate at $\mathrm{R}_{1}=0.2, \mathrm{ASR}=10$, and $\mathrm{Bi}=$ 0.12 at different values of $\mathrm{F}_{0}$

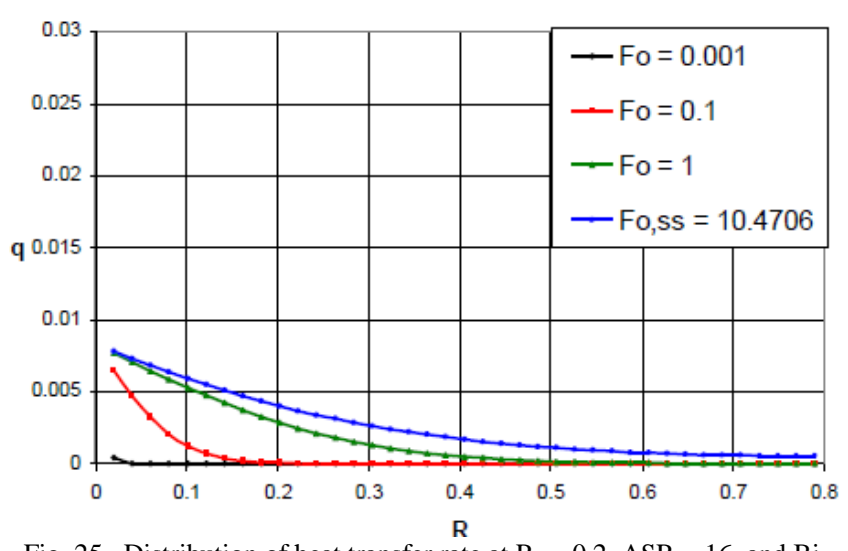

Fig. 25 Distribution of heat transfer rate at $\mathrm{R}_{1}=0.2, \mathrm{ASR}=16$, and $\mathrm{Bi}=$ 0.12 at different values of $\mathrm{F}_{0}$ 


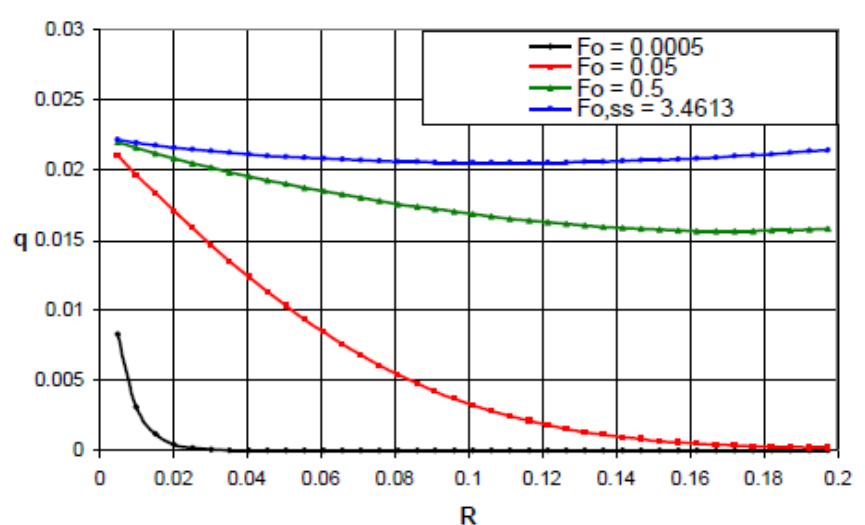

Fig. 26 Distribution of heat transfer rate at $\mathbf{R}_{\mathbf{1}}=\mathbf{0 . 8}, \mathbf{A S R}=\mathbf{1 0}$, and $\mathbf{B i}=$ $\mathbf{0 . 1 2}$ at different values of $\mathbf{F}_{\mathbf{o}}$

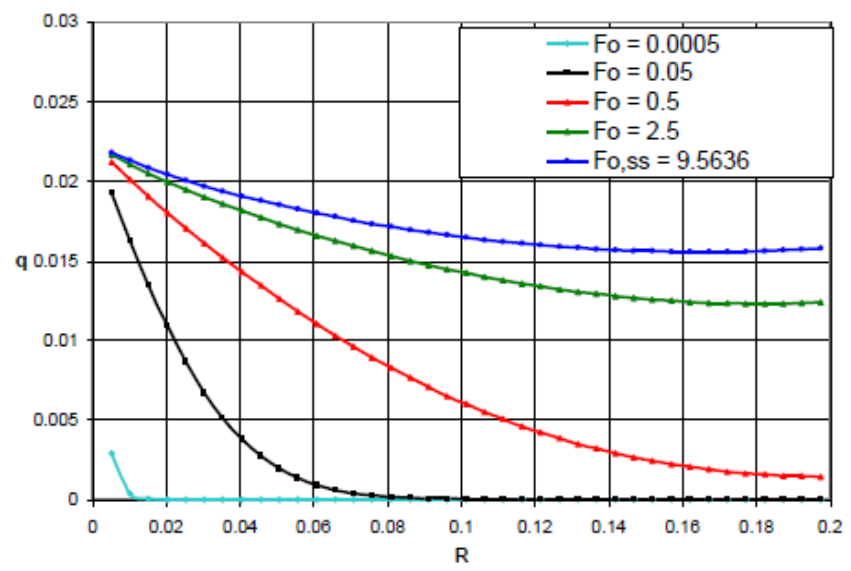

Fig. 27 Distribution of heat transfer rate at $\mathrm{R}_{1}=0.8, \mathrm{ASR}=16$, and $\mathrm{Bi}=$ 0.12 at different values of $F_{o}$

\section{Temperature Contour Lines}

In this section, the mechanism of heat conduction through the fin material is presented in qualitative figures under steady state conditions. These figures in general show the path that the heat is selecting through the fin material before it reaches the surface and therefore convected by the surrounding fluid. For this purpose, eight geometries representing the limiting cases of $\boldsymbol{B i}=\mathbf{0 . 0 4}$ and $\mathbf{0 . 1 2}, \boldsymbol{A S R}=$ 10 and $\mathbf{1 6}$, and $R_{1}=\mathbf{0 . 2}$ and $\mathbf{0 . 8}$. The contour lines of the temperature for all cases start at a value of unity at the fin base and decreases as we move away towards the fin tip. In general, the temperature contour lines show the direction of heat flow and its magnitude. The flow of heat takes paths that are orthogonal to the temperature contour lines. The rate of heat transfer increases as the temperature gradient increases. This can be read by the degree of change in the value of the temperature from one contour line to the next ones. As the changes are larger, the heat transfer rate is also larger and vice versa.

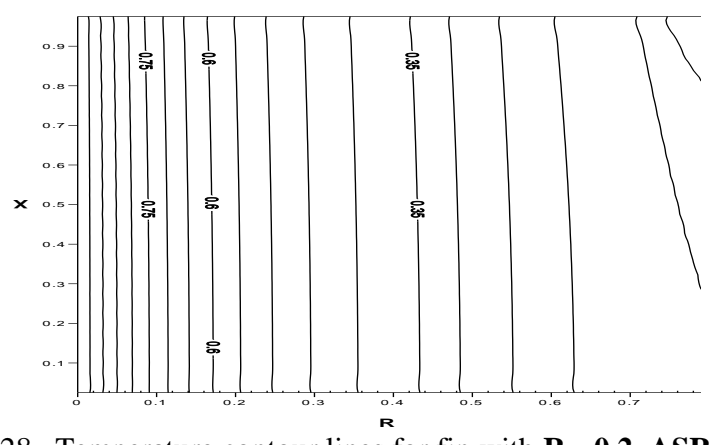

Fig. 28 Temperature contour lines for fin with $\mathbf{R}_{\mathbf{1}}=\mathbf{0 . 2}, \mathbf{A S R}=\mathbf{1 0}$, and $\mathbf{B i}=\mathbf{0 . 0 4}$

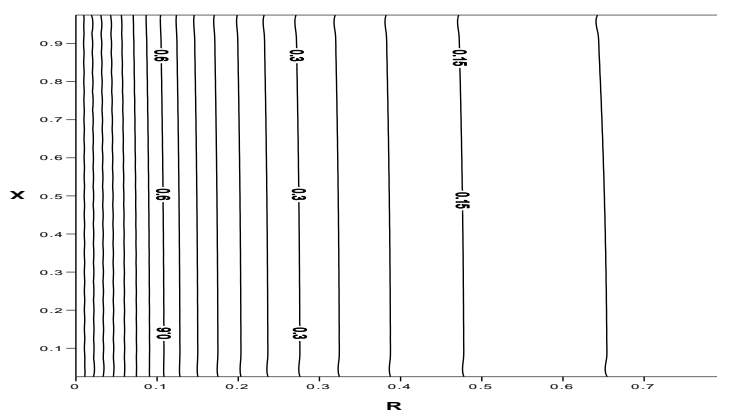

Fig. 29 Temperature contour lines for fin with $\mathrm{R}_{1}=0.2, \mathrm{ASR}=16$, and $\mathrm{Bi}=0.04$

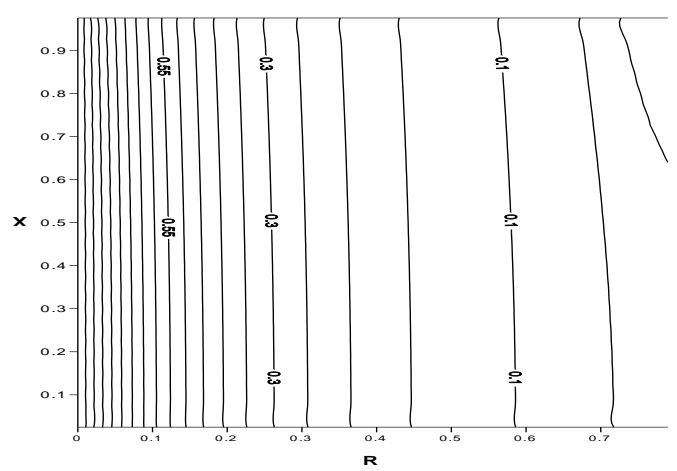

Fig. 30 Temperature contour lines for fin with $\mathrm{R}_{1}=0.2$, ASR $=10$, and $\mathrm{Bi}=0.12$

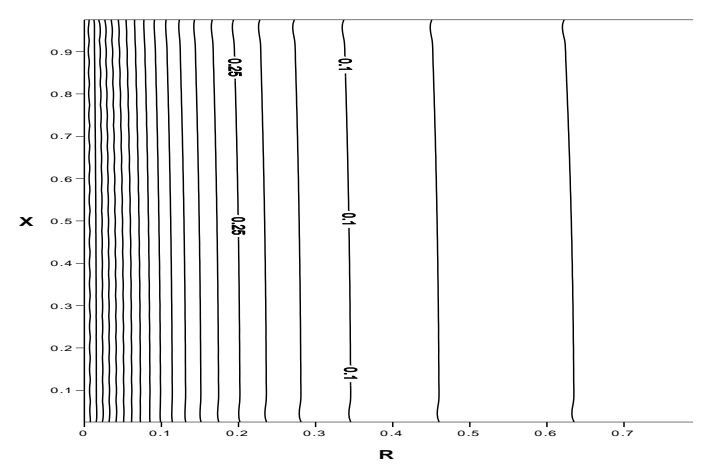

Fig. 31 Temperature contour lines for fin with $\mathbf{R}_{\mathbf{1}}=\mathbf{0 . 2}, \mathbf{A S R}=\mathbf{1 6}$, and $\mathbf{B i}=\mathbf{0 . 1 2}$ 


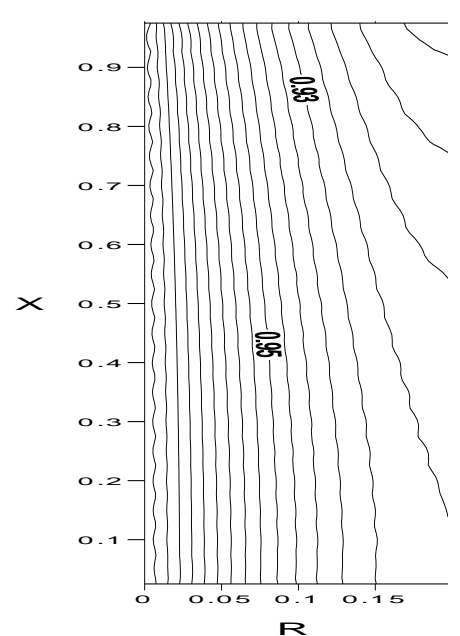

Fig. 32 Temperature contour lines for fin with $\mathrm{R}_{1}=0.8$, $\mathrm{ASR}=10$, and $\mathrm{Bi}=0.04$

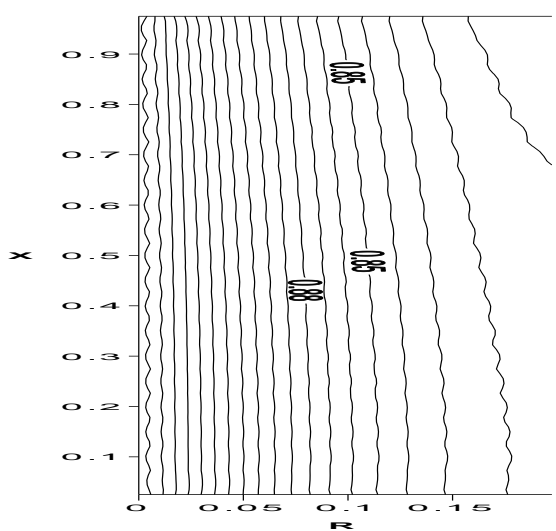

Fig. 33 Temperature contour lines for fin with $\mathrm{R}_{1}=0.8, \mathrm{ASR}=16$, and $\mathrm{Bi}=0.04$

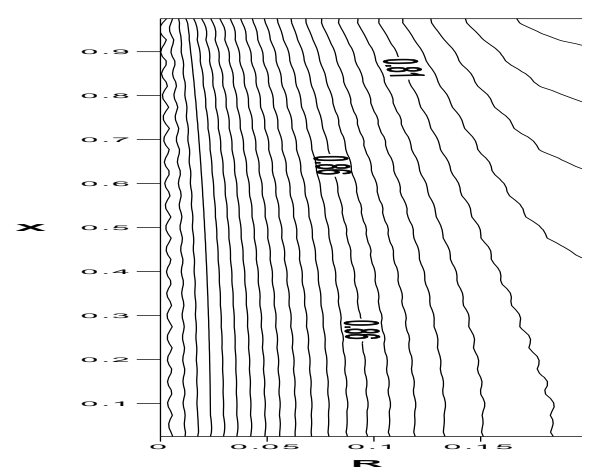

Fig. 34 Temperature contour lines for fin with $\mathrm{R}_{1}=0.8$, $\mathrm{ASR}=10$, and $\mathrm{Bi}=0.12$

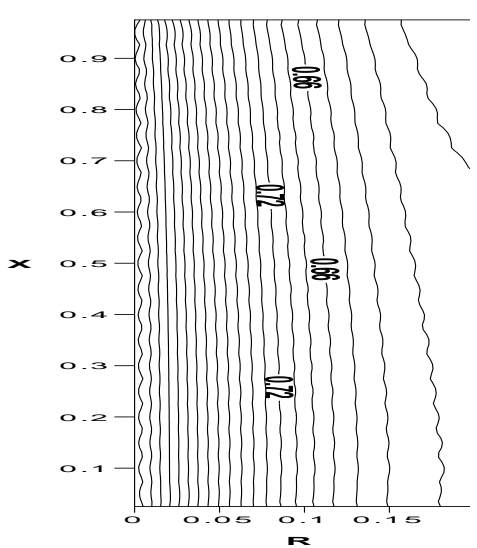

Fig. 35 Temperature contour lines for fin with $\mathbf{R}_{\mathbf{1}}=\mathbf{0 . 8}, \mathbf{A S R}=\mathbf{1 6}$, and $B \mathbf{i}=\mathbf{0 . 1 2}$

\section{CONCLUSIONS}

A numerical analysis of the annular fin was carried out for different geometrical and operational parameters. The effect of varying of $\boldsymbol{A S R}, \boldsymbol{R} \boldsymbol{1}$, and $\boldsymbol{B i}$ were presented and discussed. The results presented in the previous chapter can lead to the following conclusions:

1. The efficiency of the fin decreases as $\boldsymbol{B} \boldsymbol{i}$ increases, and the rate of decrease is sharp at lower values of $\boldsymbol{B i}$ while it becomes smaller by increasing of $\boldsymbol{B i}$.

2. The values of the efficiency at any $\boldsymbol{B} \boldsymbol{i}$ are shown to be higher at higher values of $\boldsymbol{R}_{\boldsymbol{I}}$ and consistently lower at higher values of $\boldsymbol{A S R}$.

3. The heat transfer rate decreases as $\boldsymbol{B} \boldsymbol{i}$ increases. Also, it increases by increasing $\boldsymbol{R} \boldsymbol{l}$ at all values of $\boldsymbol{B i}$ and $\boldsymbol{A S R}$, however the effect of $\boldsymbol{A S R}$ is shown to have the opposite effect of decreasing the heat transfer rate at all values of $\boldsymbol{B i}$ and $\boldsymbol{R} \boldsymbol{l}$.

4. Thick and short fins (with minimum $\boldsymbol{A S R}$ and maximum $\boldsymbol{R}_{\boldsymbol{1}}$ ) are found to have better performance than thin and long ones (with maximum $\boldsymbol{A S R}$ and minimum $\boldsymbol{R}_{\boldsymbol{I}}$ ).

\section{REFERENCES}

[1] J P Holman, Heat Transfer, 10th ed. NewYork, McGraw-Hill, 2010.

[2] Patankar, s. v., Numerical Heat Transfer and Fluid Flow, Hemisphere Publishing Corporation, 1980

[3] Sucer, J., Heat Transfer, WM. C. Brown, 1st Edition, 1985. 